Opinión de la Junta Directiva de la Asociación de Radiólogos de Navarra ante la práctica de ecografías por médicos no radiólogos

\title{
The Board of Directors of the Radiologist Association of Navarra's statement on the utilization of ultrasound by non-radiologist physicians
}

https://doi.org/10.23938/ASSN.0387

Junta Directiva de la Asociación de Radiólogos de Navarra

\section{Sr Editor:}

En el último número de la revista Anales del Sistema Sanitario de Navarra se publicó un editorial sobre la ecografía en la práctica asistencial en Atención Primaria $^{1}$ y un artículo sobre la realización de ecografía a pie de cama en Urgencias ${ }^{2}$; desde la Junta Directiva de la Asociación de Radiólogos de Navarra (ARN) querríamos hacer unos comentarios en relación a la realización de ecografías por médicos no radiólogos.

La ecografía es una técnica diagnóstica no invasiva, ampliamente extendida en la práctica médica y que es, muchas veces, un filtro para la indicación de otras pruebas. También es una técnica operador-dependiente, cuya eficacia depende de la habilidad y de la experiencia del ecografista.

El programa formativo de la especialidad de Radiodiagnóstico ${ }^{3}$ establece que el médico residente de Radiología debe participar en la realización de, al menos, 1.600 ecografías durante su periodo formativo en las distintas áreas específicas de la Ra- diología (neurorradiología, radiología abdominal, radiología de mama, pediátrica, musculoesquelética, torácica, y vascular e intervencionista). El aprendizaje es progresivo: primero se adquieren las bases teóricas y posteriormente se realiza la técnica de forma tutelada hasta ir adquiriendo autonomía.

En los últimos años se ha extendido el uso de la ecografía entre médicos no radiólogos, y nosotros vemos esta tendencia con bastante preocupación y escepticismo, principalmente porque dichos profesionales no tienen la formación que establece el Ministerio de Sanidad, Consumo y Bienestar Social.

Es una realidad que incluso expertos especialistas en Radiología pueden tener dudas al interpretar los hallazgos de una ecografía, pero conocen las limitaciones de la técnica y cuándo deben realizarse exploraciones complementarias. Por eso, consideramos imprudente que profesionales sin la formación adecuada hagan ecografías, ya que pueden incurrir en dos riesgos: sobrediagnosticar, lo que lleva a la realización de
Recepción: 01/11/2018

Aceptación definitiva: 04/12/2018

\section{Correspondencia:}

Helena Gómez Herrero

Vicepresidenta de la Asociación de Radiólogos de Navarra

Servicio de Radiología

Complejo Hospitalario de Navarra

C/ Irunlarrea, 3

31008 Pamplona

E-mail: hgomezhe@cfnavarra.es 
otros estudios a pacientes que no lo precisarían si hubieran sido correctamente valorados $\mathrm{y}$, mucho peor, a diagnosticar falsos negativos de patologías debido a la escasa formación.

Asimismo, no consideramos procedente que se realice una prueba médica como es la ecografía sin emitir informe, como apreciamos en nuestra práctica habitual, cuando el paciente viene a realizarse una ecografía a un servicio de Radiología y nos refiere que otro médico ya le ha realizado antes la misma prueba, de la que no hay constancia escrita. En contraposición, el radiólogo siempre emite un informe de la prueba que realiza como acto médico que es y como criterio de calidad de la misma ${ }^{4}$.

Por otra parte, el gasto en ecógrafos para las distintas especialidades es especialmente agraviante si no se garantiza su uso suficiente, ya que en los servicios de Radiología disponemos actualmente de algunos aparatos a pleno rendimiento que ya están obsoletos. Consideramos que el radiólogo, como especialista de imagen, debe disponer de la mejor tecnología.

Los radiólogos, al estar también involucrados en el proceso de diagnóstico, tratamiento y seguimiento de los pacientes, mantenemos una estrecha relación con la mayoría de las especialidades médicas. Somos conscientes de la importancia, y también de la dificultad, que supone una buena valoración del paciente, la anamnesis, la exploración física y la valoración de otras pruebas. Nos preocupa que la realización de ecografías en su trabajo vaya en detrimento de esta valoración, bien porque impida tener tiempo suficiente o porque terminen por sustituirla por la imagen. Si después de una adecuada valoración del paciente hay que hacer una prueba de imagen, lo ideal sería que la realice el especialista formado específicamente para ello.

Somos conscientes de que la demora en la realización de las pruebas ecográficas por parte de los servicios de Radiología ha contribuido a la externalización de esta técnica, pero no estamos seguros de que, para atender esta demanda, se hayan buscado todos los recursos posibles dentro de dichos servicios.
Los radiólogos somos realistas y conocedores de que, en las circunstancias actuales, esta generalización de la ecografía por distintos especialistas es imparable. La situación actual, fruto de una decisión no consensuada con los servicios de Radiología, conlleva además cierto conflicto entre profesionales, que debería ser evitado ${ }^{5}$, al no definir exactamente la responsabilidad que corresponde a cada uno dentro del proceso asistencial.

Para no banalizar la técnica y optimizar su correcto funcionamiento en beneficio de los pacientes, pensamos que el uso de la ecografía por médicos no radiólogos debería limitarse a situaciones clínicas concretas que habría que establecer.

No se puede pretender sustituir a los radiólogos, que son especialistas específicamente formados para realización de pruebas de imagen médicas, cuya labor lleva años de preparación, por otros profesionales que han realizado algún curso o jornada sobre ecografía de unas horas de duración.

Desde la Junta Directiva de la ARN nos gustaría que se potenciase el desarrollo de la ecografía en los servicios de Radiología, pues el modelo especializado de realización de ecografías es el que disminuye los costes sanitarios ${ }^{6}$, y los radiólogos somos los especialistas que tenemos la formación para realizar esta exploración.

La SERAM (Sociedad Española de Radiología Médica) ha emitido recientemente un documento ${ }^{7}$ con propuestas concretas sobre la situación actual de la ecografía que buscan mejorar la atención sanitaria, y que esperamos que sean tenidas en cuenta por las diferentes administraciones y gerencias de servicios sanitarios, incluidas las de nuestra comunidad.

\section{BIBLIOGRAFÍA}

1. Malón Musgo MM. Ecografía en la práctica asistencial en Atención Primaria. An Sist Sanit Navar 2018; 41: 157-160. https://doi. org/10.23938/ASSN.0313

2. Jacob J, Zorrilla J, Gené E, Alonso G, Rimbau $\mathrm{P}$, Casarramona $\mathrm{F}$ et al. Análisis del uso de la ecografía a pie de cama en los servicios de 
urgencias hospitalarios de Cataluña. Estudio ECURCAT. An Sist Sanit Navar 2018; 41: 161169. https://doi.org/10.23938/ASSN.0300

3. Ministerio de Sanidad y Consumo. Orden SCO/634/2008 por la que se aprueba y publica el programa formativo de la especialidad de Radiodiagnóstico. BOE 60, de 10 de marzo de 2008: 14333-14341.

4. Morales A, del Cura JL, Bueno A, Remartínez E, García P, Méndez S et al. Documentos SEUS 2013-03. El informe en ecografía. Madrid: Sociedad Española de Ultrasonidos; 2013.

5. Herranz Rodríguez G, Muñoz Fronteras R. Las fronteras internas del ejercicio profesional. Declaración de la Comisión Central de Deontología. Organización Médica Colegial de España; 2007. https://www.cgcom.es/sites/ default/files/fronteras_internas_ejercicio_ profesional.pdf

6. Morales Santos A, Del Cura Rodríguez JL, Nicolau Molina C, Remartínez Escobar E, Bueno Horcajadas A, García González P. Documentos SEUS 1-2012. Los costes de la ecografía. Madrid: Sociedad Española de Ultrasonidos; 2012. https://www.seram.es/images/site/ seus-sociedad-espa\%C3\%B1ola-de-ultrasonidos.original.pdf

7. Sociedad Española de Radiología Médica. Posicionamiento de la SERAM sobre la ecografía en atención primaria. https://seram. es/index.php/informacion/noticias/1206-posicionamiento-de-la-seram-sobre-la-ecografia-en-atencion-primaria. Publicado el 11 de noviembre de 2018. 
